\title{
"Cannot intubate, cannot ventilate" scenario and failed tracheostomy in a patient with massive neck swelling: a case report
}

\begin{abstract}
Introduction: Neck swelling associated with airway obstruction is a significant challenge in airway management. When the neck swelling is very large and the airway anatomy is grossly distorted, even surgical tracheostomy may prove impossible.

Case report: We present a case of massive neck swelling causing airway compromise, complicated by difficult ventilation, intubation and failed tracheostomy. A 53-year-old woman presented with a submandibular swelling, diagnosed as Ludwig's angina. However she refused surgical intervention and only agreed to antibiotics. Six weeks later, she came in again with airway compromise. Endotracheal intubation was attempted in the operating theatre with otorhinolaryngology (ENT) surgeon backup. Intubation was difficult by direct laryngoscope and "Glidescope" video laryngoscope. Mask-ventilation and Proseal ventilation later became impossible. Emergency tracheostomy also failed because the trachea could not be located. She developed hypoxic cardiac arrest.

Conclusion: Acute airway obstruction from massive neck swelling should be jointly managed by the anaesthetist, surgeon and a well-informed team in the operating theatre. "Conscious sedation" using dexmedetomidine or remifentanil infusion may allow the use of fibreoptic intubation in un-cooperative patients.
\end{abstract}

Keywords: Neck swelling; Airway obstruction; Difficult intubation; Failed tracheostomy
Volume 2 Issue 5 - 2015

\section{Chin KH, Wael HM, Kamisah MT}

Department of Anaesthesia \& Intensive Care, Malaysia

\begin{abstract}
Correspondence: Chin Kim Hong, Department of Anaesthesia \& Intensive Care, Hospital Sultanah Nora Ismail, Jalan Korma, 83000, Batu Pahat, Johor, Malaysia ext. 4339, Tel +607-4363000; Email acchill_chin@hotmail.com
\end{abstract}

Received: May 19, 2015 | Published: August 06, 2015

\section{Introduction}

Neck swellings are a great concern to patients and healthcare providers. Patients can present with disabling symptoms, such as dysphagia, odynophagia, obstructive sleep apnoea, stridor and respiratory distress. Depending on the origin of the swelling, some can even cause metabolic changes. Goitre with hyperthyroidism can manifest with tachycardia, hypertension, restlessness and heat intolerance. Abscess collection causes pain, local lymphadenopathy and systemic inflammatory response syndrome.

Neck swellings can cause trismus, limited head and neck movements, elevation of the tongue, fragile mucosa, bleeding and airway stenosis, making airway management a great challenge. In the 4th National Audit Project (NAP4) on major airway complications by the Royal College of Anaesthetists and the Difficult Airway Society, head and neck pathologies account for $40 \%$ (72 cases) of the total cases reported (184 cases). About $70 \%$ of these cases involved airway obstruction. The outcome of these cases were poor, including death or brain damage in 13 cases, emergency surgical airway in 50 cases and unexpected ICU admission in $27 .{ }^{1}$

There is no consensus on the ideal method of airway management in these patients. Evidence came in the form of case reports and small studies, with many confounding factors. The accepted methods include inhalational induction, awake fibreoptic intubation (AFOI) or tracheostomy under local anaesthesia. ${ }^{2}$ In partial airway obstruction, patients are depending on awake muscle tone to keep their airway patent. Many anaesthetists advocate to preserve spontaneous respiration until a definitive airway is secured, therefore avoiding intravenous induction agent and muscle relaxant. The final decision depends on many factors, including the urgency of intubation, the nature of the neck swelling, patient's cooperation, presence of respiratory distress, other co-morbidities, the experience of the anaesthetist, surgical expertise, availability of equipments and location. ${ }^{2}$ The primary plan should be backup by a plan $\mathrm{B}$, or even $\mathrm{C}$ and $\mathrm{D}$, because any plan can fail and these patients deteriorate very rapidly.

\section{Case Report}

A 53-year-old lady presented to our emergency department with a neck swelling, associated with difficulty swallowing. Two weeks before, she had a toothache and attempted to extract her left molar tooth by herself. However only part of the tooth came out. She did not seek medical treatment at the time. Her left submandibular region became progressively swollen, restricting her mouth opening and oral intake. She went to a traditional medicine practitioner, but to no avail. She had no known medical illness and weighed $60 \mathrm{~kg}$.

She was breathing comfortably without stridor or wheeze. The neck swelling measured $10 \times 10 \mathrm{~cm}$, extending from the left submandibular region to the anterior midline, displacing the trachea towards the right side. It was firm, erythematous, tender and non-fluctuant. Her mouth opening was restricted with an inter-incisor distance of $2 \mathrm{~cm}$ only. The floor of her mouth was raised on the left side, with pus discharging from the left buccal sulcus. The oropharynx could not be visualised, Mallampati score IV. Her full blood count revealed a total white count of $11.7 \times 103 / \mu \mathrm{L}$ ( $81 \%$ granulocytes), haemoglobin of $14.4 \mathrm{~g} / \mathrm{dL}$ and platelet count of $201 \times 103 / \mu \mathrm{L}$ (Figure 1).

An urgent contrast-enhanced computed tomography (CT) scan revealed a left submandibular space mass measuring $7.5 \times 13.1 \mathrm{x}$ $12.2 \mathrm{~cm}$, with bilateral cervical lymphadenopathy and extensive infiltration into the left parotid gland, left pterygoid muscles, left submandibular gland and left sternocleidomastoid muscle. The trachea, cricoid cartilage, thyroid gland, hyoid bone, oro- and hypopharynx were displaced to the contralateral side. The differential diagnoses included lymphoma or non-liquefied abscess (Figure 2). 


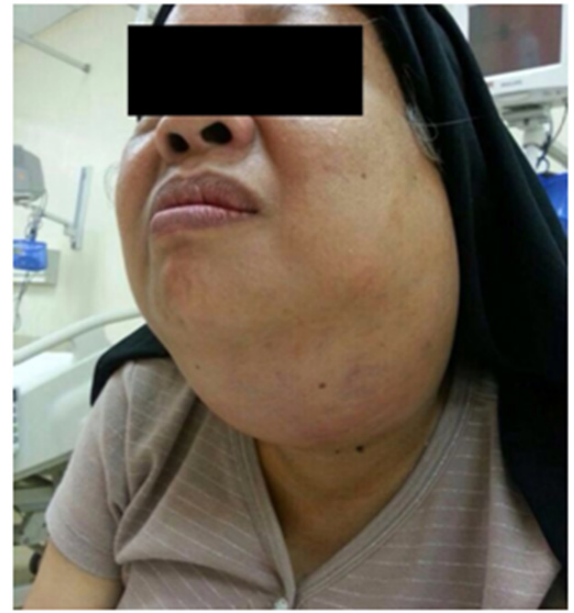

Figure I Patient during First Admission.

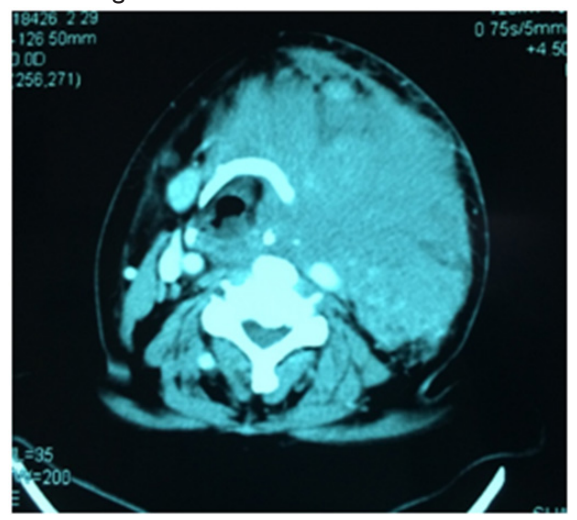

Figure 2 Cervical CT Scan Showed a Large Mass Displacing the Midline Structures to the Right Side.

She was diagnosed as having Ludwig's angina secondary to dentoalveolar abscess and was given intravenous co-amoxiclav and metronidazole. She refused all invasive procedures, including nasal endoscopy, biopsy and tooth extraction, despite elaborated explanation by the ENT surgeon, maxillofacial surgeon and anaesthetist. After 3 days in the ward, she even refused intravenous cannulation. She was put on oral medications and was discharged from the ward with follow-up appointments, which she defaulted.

Six weeks later she came in again with shortness of breath and a worsening dysphagia. The neck swelling has become larger $(20 \mathrm{x}$ $15 \mathrm{~cm}$ ), extending to the left infra-orbital and right submandibular regions. She appeared lethargic and tachypnoeic. She could not talk, responding to questions by head and hand gestures only. After stabilisation, an urgent nasal endoscopy was done. The epiglottis was oedematous, but the vocal cords could not be visualised. In view of the compromised airway and respiratory distress, we decided for endotracheal intubation (Figure 3).

She was reviewed by the anaesthetist at the emergency department. Anticipating a difficult airway, intubation was attempted in the operating theatre with ENT surgeon and theatre staff scrubbedup and standby for emergency tracheostomy. The patient had become more tachypnoeic and restless by then. She was induced with sevoflurance in 100\% oxygen. Direct laryngoscopy could not visualise the epiglottis (Cormack-Lehane grade IV). Glidescope video laryngoscope also encountered difficulty because the pharynx was friable and bled easily on contact. The bleeding repeatedly obscured our view. A swollen, anteriorly-displaced epiglottis was seen during the second Glidescope attempt, but intubation was unsuccessful. Subsequently mask ventilation became impossible. A Proseal was inserted to assist ventilation but the tidal volume generated was still low. Patient's oxygen saturation began to drop. A dose of intravenous suxamethonium $1 \mathrm{mg} / \mathrm{kg}$ was given to abolish any laryngospasm and to improve ventilation. Emergency surgical tracheostomy was attempted but the trachea could not be identified. The oxygen saturation dropped further and the patient developed hypoxic cardiac arrest. Cardiopulmonary resuscitation was performed for 2 minutes before the return of spontaneous circulation. A last-ditch effort was made with the Glidescope and intubation was finally successful with an ID $6.0 \mathrm{~mm}$ endotracheal tube. The oxygen saturation and ventilation improved. Tracheostomy was aborted due to the grossly distorted airway anatomy (Figure 4).

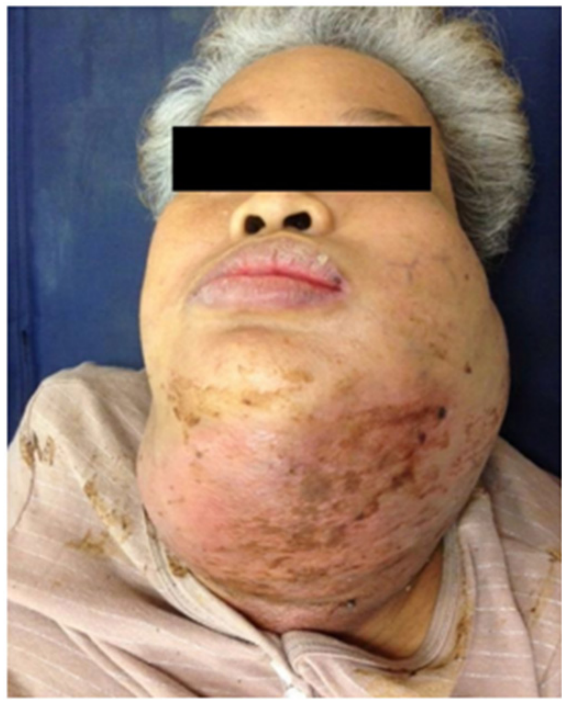

Figure 3 Patient during Second Admission.

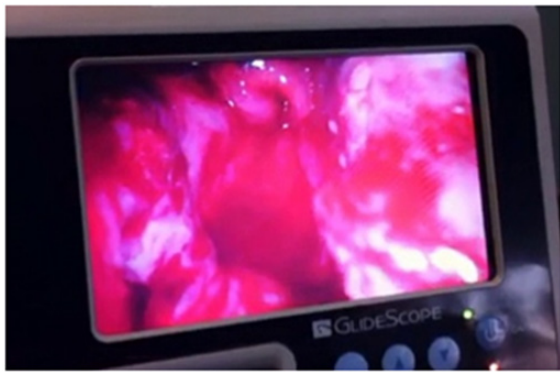

Figure 4 Glidescope Showing an Oedematous Epiglottis and a Friable Mucosa with Contact Bleeding.

The patient had severe metabolic acidosis post-cardiac arrest and developed unstable ventricular tachycardia. She was cardioverted, and given intravenous amiodarone infusion and sodium bicarbonate. Her blood pressure remained low despite starting noradrenaline and dobutamine infusions. She passed away after a second episode of cardiac arrest the next day.

\section{Discussion}

The management of airway obstruction caused by neck swelling should be a team effort, as demonstrated in this case. The airway management plans were discussed and agreed by both the anaesthetist and the surgeon, and conveyed clearly to the other team members. The surgeon and theatre staff had scrubbed-in and standby when intubation attempt began. These are in accordance to the recommendation by the NAP4. 
As mentioned above, the airway management options for this patient include inhalational induction, awake fibreoptic intubation and tracheostomy under local anaesthesia. She was tachypnoeic, restless and deemed uncooperative for AFOI. In the process of securing the airway, both inhalational induction and tracheostomy failed and patient deteriorated into a "cannot intubate, cannot ventilate" scenario. It is evident that any plan may fail, and a good backup plan (or plans) must be prepared in advance. The theoretical advantages of inhalational induction are the preservation of spontaneous respiration and protective airway tone. Theoretically if intubation failed, we can maintain ventilation and awake the patient. In a critical airway, volatile agents can significantly reduce respiratory drive and cause airway collapse. In this case, the patient's spontaneous tidal volume dropped rapidly with sevoflurane induction and required positive pressure ventilation to achieve adequate depth of anaesthesia for airway manipulation. The initial advantage of maintaining spontaneous respiration is lost. IF we decided not to deepen the anaesthesia and wake her up, she may not tolerate AFOI or tracheostomy under local anaesthesia in view of her respiratory distress and agitation.

After 2 failed attempts of intubation, ventilation became difficult probably due to laryngospasm. A dose of suxamethonium was given to improve ventilation and intubating condition. This is a debatable decision. Some authors propose that muscle relaxant can abolish the protective airway tone and cause sudden total obstruction, ${ }^{3}$ but on the other hand muscle relaxant has been shown to improve mask ventilation ${ }^{4}$ and intubating condition. In the management of airway obstruction causing hypoxia, the NAP4 recommends giving a dose of muscle relaxant to assist ventilation and intubation before the patient deteriorat to the stage where an emergency surgical airway is necessary. ${ }^{1}$

Tracheostomy under local anaesthesia was not an option in this case in view of grossly distorted anatomy and restless patient. Even under general anaesthesia, tracheostomy was unsuccessful. The trachea could not be identified and the ENT surgeon had to abort the surgery in the end. Even the backup plan has failed. The same can be said for cricothyroidotomy the anatomy is very distorted the surgeon can't find the trachea or even the larynex.

AFOI is a valuable tool in the management of supraglottic airway obstruction from head and neck swelling. The flexible scope can be maneuvered around the swelling to identify laryngeal structures. Even if it fails the patient remains awake and breathing normally. We did not attempt AFOI as the patient was restless and tachypnoeic. She was unable to stay still. Furthermore the administration of local anaesthetic to the inflamed, oedematous mucosa will probably not achieve good anaesthesia. A possible solution may be fibreoptic intubation under sedation. Conventional sedating agents, such as benzodiazepines, opioids and propofol, can cause respiratory depression and airway compromise. Ketamine increases airway secretion. Dexmedetomidine and remifentanil are 2 agents that produce "conscious sedation" and analgesia. The selective alpha2-agonist, dexmedetomidine, has been shown to be superior to sufentanil ${ }^{5}$ and propofol target- controlled infusion (TCI) ${ }^{6}$ in nasotracheal intubating condition and patient tolerance. It also has a lower incidence of hypertension and desaturation. Remifentanil, administered by a TCI device, offers stepwise titration to achieve sedation while maintaining response to verbal command. ${ }^{7}$ The short context-sensitive half time of remifentanil and the availability of an antagonist improve patient safety. The studies quoted above have small sample sizes of $<50$ patients, therefore more investigations are needed. Both dexmedetomidine and remifentanil are not available in our hospital setting.

\section{Conclusion}

Patients with acute airway obstruction caused by massive neck swellings should be jointly managed by the anaesthetists and surgeons. The management plan must include backup plans because any plan may fail, as demonstrated in this case. Intubation is best carried out in the operating theatre, with the surgeon scrubbed-up and ready to perform emergency tracheostomy should the need arise. Fibreoptic intubation under "conscious sedation" may be a valuable option in the management of difficult airway in un-cooperative patients.

\section{Acknowledgments}

None.

\section{Conflicts of Interset}

None.

\section{References}

1. Cook T, Woodall N, Frerk C(2011) Major complications of airway management in the UK: results of the 4th National Audit Project of the Royal College of Anaesthetists and the Difficult Airway Society. Br J Anaesth 106:617-631.

2. Patel A and Pearce A (2011) Progress in Management of the Obstructed Airway Anaesthesia 66(Suppi 2):93-100.

3. Mason RA, Fielder CP (1999) The Obstructed Airway in Head and Neck Surgery. Anaesthesia 54(7):625-628.

4. Waters RD, Szabo TA, Spinale FG, Desantis SM, Reves JG (2011) The Effect of Neuromuscular Blockade on Mask Ventilation Anaesthesia 66(3): 163-167.

5. Shen SL, Xie YH, Wang WH, Hu SF, Zhang YL (2014) Comparison of Dexmedetomidine and Sufentail for Conscious Sedation in Patients Undergoing Awake Fibreoptic Nasotracheal Intubation: a Prospective, Randomised and Controlled Clinical Trial. Clin Respir J 8(1):100-107.

6. Tsai CJ, Chu KS, Chen TI, Lu DV, Wang HM, et al. (2010) A comparison of the Effectiveness of Dexmedetomidine Versus Propofal Target-controlled Infusion for Sedation during Fibreoptic Nasotracheal Intubation Anaesthesia 65(3):254-259.

7. Vennilla R, Hall A, Ali M, Bhulyan N, Pirotta D, et al. (2011) Remifentanil as Single Agent to Faciliate Awake Fibreoptic Intubation in the Absence Of Premedication Anaesthesia 66(5):368-372. 